thebmj

\title{
Editorials
}

\section{Vitamin A supplements and survival in children}

BMJ 2010; 340 doi: http://dx.doi.org/10.1136/bmj.c977 (Published 09 March 2010) Cite this as: BMJ 2010;340:c977

\section{Andrew M Prentice, professor of international nutrition \\ ${ }^{1}$ MRC International Nutrition Group, London School of Hygiene and Tropical Medicine, London WC1N 4AL and MRC Keneba, Gambia}

andrew.prentice@Ishtm.ac.uk

New evidence points to a differential effect in girls and boys

In the linked randomised controlled trial (doi:10.1136/bmj.c1101), Benn and colleagues assess the effect of giving high dose vitamin A supplements to low birthweight neonates in Guinea-Bissau. Overall, they found no effect on infant mortality, although the effect differed by sex; boys tended to benefit but a significantly harmful effect was seen on girls' survival.1

In 1983 it was reported that young Indonesian children with mild xerophthalmia, Bitot's spots, and night blindness - the clinical symptoms of vitamin A deficiency-had a higher risk of death.2 A subsequent randomised controlled trial of vitamin A supplementation showed an impressive benefit on all cause mortality. 3 This prompted further large scale trials elsewhere in Asia and Africa that confirmed the effect. A meta-analysis indicated that this cheap and simple intervention reduces child mortality by $30 \%$ in countries with evidence of at least marginal vitamin A deficiency.4 The World Health Organization subsequently recommended a protocol for universal vitamin A supplementation of children aged six to 60 months, and this has been adopted as government policy in more than 60 countries worldwide.

If vitamin A supplementation is so effective at saving lives in later infancy and childhood, why not give supplements to newborn babies? Infant mortality is greatest in the first six months of life so it would be reasonable to expect an even greater benefit. The initial strategy was to supplement mothers postpartum in the hope that the benefit would be transferred through breast milk, but subsequent evidence questioned the efficacy of this approach. Safety concerns also surfaced after reports of acute bulging fontanelle after dosing, and it took some years and a WHO multicentre trial of vitamin $\mathrm{A}$, administered when the neonate is given the diphtheria-pertussis-tetanus vaccine at 2,3 , and 4 months for these anxieties to subside. 5 This paved the way for trials of neonatal vitamin A supplementation. Six such trials with reasonable power to detect effects on mortality were conducted. In 2008, WHO commissioned a meta-analysis of these trials and convened an expert advisory group to consider whether neonatal vitamin A supplementation should be adopted as policy. The meta-analysis found no survival benefit, but significant heterogeneity existed, with evidence of benefit from Asian trials and evidence of no effect (or harm) in two African trials. 6 One of the African trials that showed a trend towards increased mortality after neonatal vitamin A supplementation was conducted by Benn and colleagues in Guinea-Bissau.7 
Such a result was obviously unpalatable to the international vitamin A community, which had saved many thousands of lives through their advocacy and implementation of universal vitamin A supplementation. The finger was pointed at the fact that the Guinea-Bissau trial had intentionally excluded low birthweight babies. Surely the trial would have shown benefit if these most vulnerable neonates had been included? Sadly not. The current study by Benn and colleagues assesses neonatal vitamin A supplementation in 1717 low birthweight babies $(<2500 \mathrm{~g}$ ) born at the national hospital.1 In a two by two factorial trial, babies were randomised to receive $25000 \mathrm{IU}$ vitamin A or placebo, and early BCG or the usual later BCG. The timing of BCG had no detectable effect on mortality, and-contrary to the authors' initial expectationthey found no interaction between vitamin A and early BCG. The results for the two BCG arms were therefore combined in the analysis of the effect of supplementation.

The overall mortality rate ratio (MRR) for neonatal vitamin A supplementation versus placebo assessed up to 12 months was 1.08 (95\% confidence interval 0.79 to 1.47 ), but a significant interaction with sex was seen. The MRR was 0.74 (0.45 to 1.22$)$ in boys and 1.42 (0.94 to 2.15$)$ in girls. These results were similar to the previous trial in Guinea-Bissau in normal birthweight babies.7 The authors now present a combined analysis which yields the following MRRs: overall 1.08 (0.87 to 1.33), boys 0.80 (0.58 to 1.10), and girls 1.41 (1.04 to 1.90), with a significant interaction $(P<0.01)$ between neonatal vitamin $A$ supplementation and sex.

The post hoc combination of two trials in the absence of a formal systematic review and meta-analysis is normally discouraged and must always be interpreted with caution. However, these two trials showed remarkable homogeneity, and they independently showed a tendency towards a harmful effect of vitamin A at birth that seemed to be confined to girls. In this case a combined analysis can be defended on the grounds that the two trials represent complementary arms covering the full birthweight spectrum within the same urban population of Guinea-Bissau, and, most importantly, the latest trial was called for by international experts who predicted a beneficial effect.

The authors have a reputation for challenging dogma in relation to vaccine and micronutrient supplementation policies. Through retrospective analysis of numerous datasets (their own and those of others) they have shown repeated examples of how vaccines, micronutrients, and exposure to infections can strongly affect all cause mortality in regions with a high burden of infection. In relation to the present context, Benn and colleagues have previously reported that vitamin A and DTP (possibly all killed vaccines) can have malign effects in girls that may be potentiated when the two are combined.8 9 Sceptics have argued that their evidence has been based on "unintended experiments," and that the trends are often non-significant in isolation. The emergence of supporting evidence from prospective randomised controlled trials now cannot be ignored.

Sex differential effects, presumably relating to immune modulation, are certainly plausible. Sex differences in susceptibility to certain infections and in responses to vaccination have been recognised for decades.

Where do we go from here? With the support of the Bill and Melinda Gates Foundation, WHO has commissioned three large trials in an attempt to resolve the possibility that neonatal vitamin $A$ supplementation may be beneficial in Asia but not in Africa. As commissioned, these trials are only required to assess mortality up to six months of age. Benn and colleagues rightly argue that failure to monitor mortality until 12 months would be a costly missed opportunity and may be dangerously misleading. If, as hypothesised, the potentially negative effects of early vitamin A supplementation in girls 
are potentiated by DTP, then a longer follow-up is essential. In Benn and colleagues' combined analysis, the detrimental effect of neonatal vitamin A supplementation in girls had a MRR of 1.21 from birth to three months but 1.70 (1.08 to 2.67 ) between four and 12 months.1 Although the new trials are not individually powered to assess sex differential effects, they could help to resolve this controversy once and for all.

\section{Notes}

Cite this as: BMJ 2010;340:c977

\section{Footnotes}

\section{- Research, doi:10.1136/bmj.c1101}

- The author has completed the Unified Competing Interest form at www.icmje.org/coi_disclosure.pdf (available on request from the corresponding author) and declares that: (1) He has grant support from WHO and the Bill and Melinda Gates Foundation for work allied to the topic of this editorial; (2) $\mathrm{He}$ has had no relationships with any commercial companies that might have an interest in the submitted work in the previous three years; (3) His partner and children have no financial relationships that may be relevant to the submitted work; and (4) He has no non-financial interests that may be relevant to the submitted work."

- Provenance and peer review: Commissioned; not externally peer reviewed.

\section{References}

1. Benn CS, Fisker AB, Napirna BM, Roth A, Diness BR, Lausch KR, et al. Vitamin A supplementation and BCG vaccination at birth in low birthweight neonates: two by two factorial randomised controlled trial.

BMJ2010;340:c1101.

2. Sommer A, Tarwotjo I, Hussaini G, Susanto D. Increased mortality in children with mild vitamin A deficiency. Lancet 1983;2:585-8.

3. Sommer A, Tarwotjo I, Djunaedi E, West KP Jr, Loeden AA, Tilden R, et al. Impact of vitamin A supplementation on childhood mortality. A randomised controlled community trial. Lancet 1986;1:1169-73.

4. Fawzi WW, Chalmers TC, Herrera MG, Mosteller F. Vitamin A supplementation and child mortality. A metaanalysis. JAMA 1993;269:898-903.

5. WHO/CHD Immunisation-Linked Vitamin A Supplementation Study Group. Randomised trial to assess benefits and safety of vitamin A supplementation linked to immunisation in early infancy. Lancet 1998;352:1257-63.

6. Gogia S, Sachdev HS. Neonatal vitamin A supplementation for prevention of mortality and morbidity in infancy: systematic review of randomised controlled trials. BMJ2009;338:b919.

7. Benn CS, Diness BR, Roth A, Nante E, Fisker AB, Lisse IM, et al. Effect of 50000 IU vitamin A given with BCG vaccine on mortality in infants in Guinea-Bissau: randomised placebo controlled trial. BMJ2008;336:1416-20.

8. Benn CS, Lund S, Fisker A, Jørgensen MJ, Aaby P. Should infant girls receive micronutrient supplementation? Int J Epidemio/2009;38:586-90.

9. Benn CS, Rodrigues A, Yazdanbakhsh M, Fisker AB, Ravn H, Whittle H, et al. The effect of high-dose vitamin A supplementation administered with BCG vaccine at birth may be modified by subsequent DTP vaccination.

Vaccine 2009;27:2891-8. 